\title{
Expression of ETS1 in gastric epithelial cells positively regulate inflammatory response in Helicobacter pylori-associated gastritis
}

\author{
Yongsheng Teng ${ }^{1}$, Baocheng Cang ${ }^{2}$, Fangyuan Mao ${ }^{1}$, Weisan Chen $\mathbb{0}^{3}$, Ping Cheng ${ }^{1}$, Liusheng Peng ${ }^{1}$, Ping Luo , \\ Dongshui Lu', Nan You', Quanming Zou' and Yuan Zhuang (id)
}

\begin{abstract}
Gastric epithelial cells (GECs) provide the first point of contact of the host by Helicobacter pylori (H. pylori), and the interaction between H. pylori and GECs plays a critical role in H. pylori-associated diseases. Aberrant expression of transcription factors (TFs) contributes to the pathogenesis of inflammatory disorders, including $\mathrm{H}$. pylori-associated gastritis. ETS (E26 transformation specific) transcription factor family is one of the largest families of evolutionarily conserved TFs, regulating critical functions during cell homeostasis. We screened ETS family gene expression in $\mathrm{H}$. pylori-infected mouse and human GECs and found that ETS1 (ETS proto-oncogene 1, transcription factor) expression was highly affected by $\mathrm{H}$. pylori infection. Then, we reported that ETS1 was induced in GECs by H. pylori via cagA activated NF-KB pathway. Notably, we demonstrated that proinflammatory cytokines IL-1 $\beta$ and TNFa have synergistic effects on ETS1 expression during H. pylori infection in an NF-kB-pathway-dependent manner. RNA-seq assay and Gene-ontology functional analysis revealed that ETS1 positively regulate inflammatory response during $\mathrm{H}$. pylori infection. Increased ETS1 is also detected in the gastric mucosa of mice and patients with $\mathrm{H}$. pylori infection. Collectively, these data showed that ETS1 may play an important role in the pathogenesis of H. pylori-associated gastritis.
\end{abstract}

\section{Introduction}

Helicobacter pylori (H. pylori) infects $~ 4.4$ billion individuals worldwide and is closely associated with chronic gastritis $^{1-3}$. Chronic gastritis induced by $H$. pylori can progress to atrophic gastritis, intestinal metaplasia, dysplasia, and ultimately gastric cancer $(\mathrm{GC})^{3}$. Gastric epithelial cells (GECs) provide the first point of contact of the host for H. pylori and the interaction between H. pylori and GECs plays a critical role in $H$. pylori-associated diseases ${ }^{3,4}$.

\footnotetext{
Correspondence: Nan You (younan330@hotmail.com) or

Quanming Zou (qmzou@tmmu.edu.cn) or

Yuan Zhuang (yuanzhuang1983@yahoo.com)

${ }^{1}$ National Engineering Research Centre of Immunological Products,

Department of Microbiology and Biochemical Pharmacy, College of Pharmacy and Laboratory Medicine, Third Military Medical University, Chongqing, China

${ }^{2}$ The 988 Hospital of PLA, Henan, Zhengzhou, China

Full list of author information is available at the end of the article.

Edited by M. Diederich
}

Transcription factors (TFs) exquisitely regulate specific gene expression in a cell and play an important role in the development and function of multicellular organisms. Aberrant expression of TFs contributes to the pathogenesis of inflammatory disorders ${ }^{5}$. Previous research has demonstrated $H$. pylori can interfere with multiple TFs in GECs, such as STAT3, NF- $\mathrm{kB}$, and $\beta$-catenin, to mediate inflammatory response ${ }^{6-8}$. Although an increasing number of TFs have been explored in the H. pylori-infected GECs, further exploration is also needed.

ETS1 (ETS proto-oncogene 1, transcription factor), a member of the ETS (E26 transformation specific) TF family, plays an important role in inflammatory disorders, such as allergic lung inflammation ${ }^{9}$, atopic dermatitis ${ }^{10}$, and systemic lupus erythematosus ${ }^{11}$. The present study focuses on ETS1 participate in inflammatory response through regulating the development and function of 
immune cells ${ }^{12,13}$. However, in addition to the immune cells, ETS1 can also be expressed or induced in multiple cell types, including endothelial cells, fibroblasts, and cancer cells ${ }^{12,13}$. In gastric mucosa, it has been reported that ETS1 isn't expressed in the normal GECs; however, ETS1 is upregulated in GC cells, and associated with tumor invasion and metastasis ${ }^{14-16}$. However, what factors affect ETS1 expression in GECs is currently not known. As $H$. pylori is one of the strongest risk factors for $\mathrm{GC}^{17,18}$, it is therefore important to explore ETS1's potential role during $H$. pylori infection and chronic gastritis.

Here, increased ETS1 is detected in the gastric mucosa of patients and mice with $H$. pylori infection, and it is induced in GECs by $H$. pylori via the cytotoxin-associated gene A (cagA)-activated NF-kB pathway. RNA-seq assay and Gene-ontology functional analysis reveal that ETS1 positively regulate inflammatory response during $H$. pylori infection. Herein, we report that ETS1 may execute proinflammatory response of GECs during $H$. pylori infection.

\section{Results}

\section{H. pylori induced ETS1 expression in GECS}

Based on HUGO Gene Nomenclature Committee (HGNC) database, 28 human ETS family genes were found (Supplementary Table 1). To explore the relationship between $H$. pylori infection and ETS family gene expression in GECs, the relative expression of these genes in public microarray data from Gene Expression Omnibus (GEO) database containing expression profile data from mouse gastric epithelial progenitor-derived cell line (mGEP) infected by two H. pylori strains (chronic atrophic gastritis (ChAG)-associated $\mathrm{Kx} 1$ and gastric cancer-associated $\mathrm{K} x 2)^{19}$ were analyzed. We found that Ets1 was the most increased ETS family genes induced by H. pylori (Fig. 1a). Curiously, we then screened the ETS family genes in $H$. pylori 11637-infected AGS cells by RNA-seq. Again, ETS1 expression was the most increased (Fig. 1b). Similarly, AGS cells infected with H. pylori 11637 or 26695 showed increased ETS1 expression (Fig. 1c-left). Notably, we found that human primary GECs $\left(\mathrm{EpCAM}^{+}\right.$) infected either H. pylori 11637 or 26695 also increased ETS1 expression (Fig. 1c-right). Furthermore, AGS cells infected with either H. pylori 11637 or 26695 increased ETS1 levels in an infection time- (Fig. 1d) and dose-dependent manner (Fig. 1e). Collectively, these findings clearly indicate that $H$. pylori infection induces ETS1 expression in GECs.

cagA activated NF-кB pathway mediates ETS1 expression

To determine whether cagA (a major virulence factor of H. pylori) contributes to ETS1 expression, we first carried out transwell infection assay. The results revealed that direct contact was required (Fig. 2a). As we all know, cagA can be injected into host cells via the type IV secretion system (T4SS). Notably, ETS1 levels only increased following infection with the H. pylori 11637 but not $\triangle$ cagA in AGS cells (Fig. 2b) or human primary GECs (Fig. 2c). To further confirm ETS1 is induced in $H$. pylori-infected GECs, we conducted the luciferase reporter assay using cells containing an ETS1-luc promoter. In contrast to the uninfected control, infection with H. pylori 11637 or 26695 significantly enhanced luciferase activity in AGS cells (Fig. 2d). Furthermore, enhanced luciferase activity was also cagA dependent (Fig. 2e). Next, we found that ETS1 expression in H. pylori-infected AGS cells was suppressed by inhibiting the NF- $\mathrm{kB}$ pathway using inhibitor BAY 11-7082 (Fig. 2f, g, SFig. 1a and b). Then, the PROMO tool V.8.3 of TRANSFAC ${ }^{20,21}$ (10 maximum matrix dissimilarity rate) showed that ETS1 promoter contains two p65 binding sites (binding site 1: GCTTTTCCCAG ( -1568 to -1558$)$; and binding site 2: GACTTTCCCGA ( -373 to 363$)$ ) (Fig. 2h). Subsequently, a ChIP assay was performed. The results revealed that the H. pylori 11637infected cells increased p65 binding to the ETS1 promoter; however, the $\triangle c a g A$ did not, and BAY 11-7082 inhibited this binding (Fig. 2i). Collectively, these results showed that $\operatorname{cagA}$ activates the NF- $\mathrm{kB}$ pathway to mediate ETS1 expression.

\section{IL-1 $\beta$ and TNFa have synergistic effects on ETS1 expression during $H$. pylori infection}

Immune cell infiltration and inflammatory cytokine production are the characteristics of $H$. pylori-infected gastric mucosa ${ }^{3}$. Previous research has showed cytokines could modulate $H$. pylori-mediated gene expression in GECs, such as IFN- $\gamma$ modulates $H$. pylori-mediated B7$\mathrm{H} 2$ downregulation $^{22}$, and IL-22 modulates $H$. pylorimediated MMP10 upregulation in $\mathrm{GECs}^{23}$. Thus, we examined whether cytokines (IFN- $\gamma$, IL-17A, IL-22, IL-6, IL-12, IL-23, IL-1 $\beta$, and TNF $\alpha$ ) could modulate ETS1 expression in GECs. We showed that IFN- $\gamma$, IL-17A, IL22, IL-6, IL-12, and IL-23 had no effect on modulating ETS1 expression during $H$. pylori infection (Fig. 3a). Notably, IL-1 $\beta$ and TNF $\alpha$ exerted a synergistic effect on H. pylori-mediated ETS1 expression (Fig. 3b-e). Then, the expression of ETS1 was suppressed by using BAY 11-7082 in IL-1 $\beta$ or TNF $\alpha$ stimulated AGS cells (Fig. 3f-i). Taken together, these findings suggest that IL-1 $\beta$ and TNF $\alpha$ have synergistic effects on $H$. pylori-mediated ETS1 expression through the NF-kB pathway.

\section{ETS1 positively regulate inflammatory response during $H$. pylori infection}

To identify ETS1-positive regulate genes during $H$. pylori infection, we first suppressed ETS1 by siRNA followed by $H$. pylori infection, and then performed RNA-seq assay. 

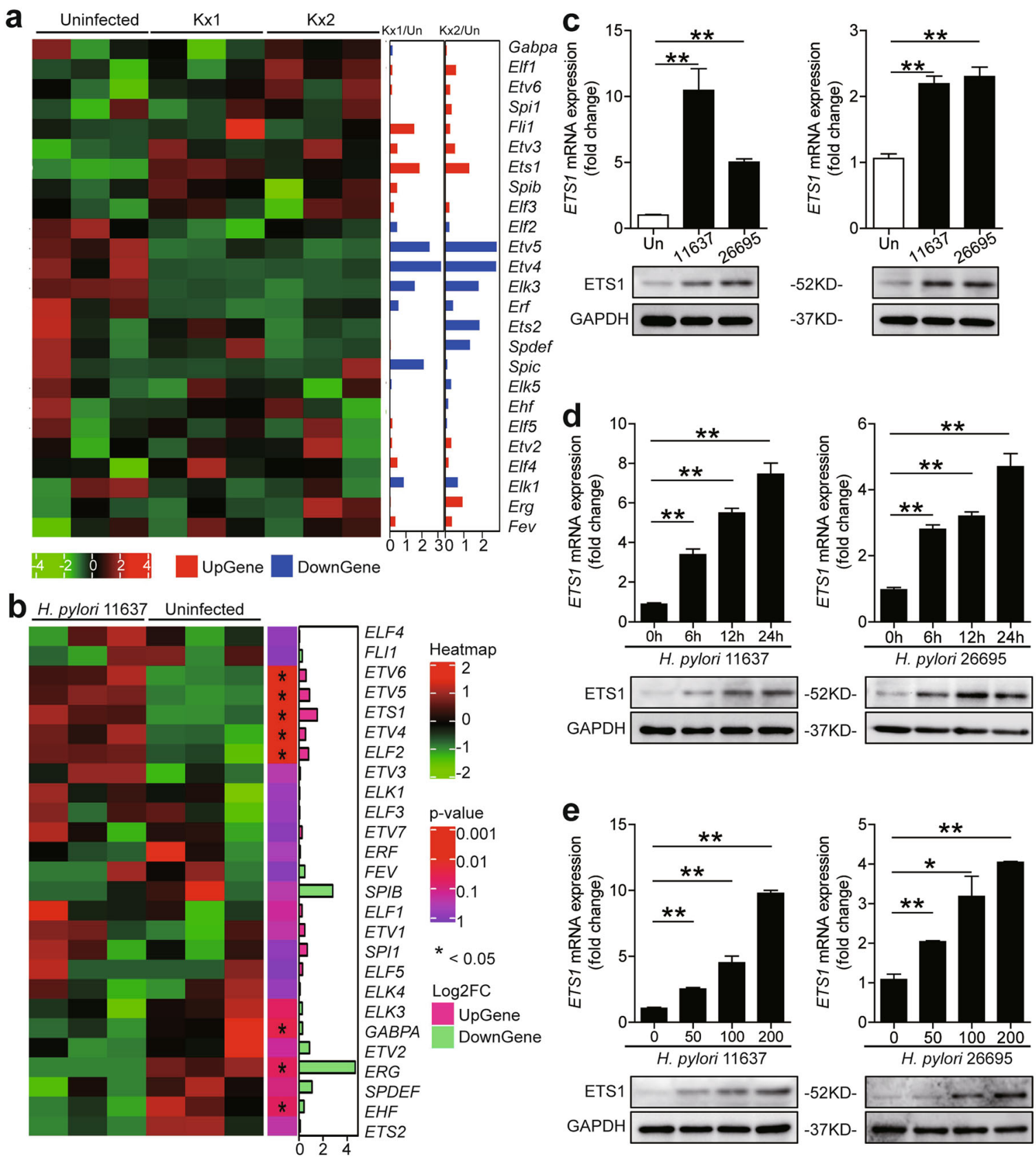

Fig. 1 H. pylori induced ETS1 expression in GECs. a Relative expression of ETS transcription factor family genes in mouse gastric epithelial progenitor-derived cell line (mGEP) infected with clinically isolated $H$. pylori strains $K x 1$ and $K \times 2$. The data were obtained from the GEO database (GSE10262). b RNA-seq analysis of expression of ETS transcription factor family genes in H. pylori 11637-infected AGS cells (ETV3L and SPIC were not detected). c ETS1 mRNA and protein expression in H. pylori 11637-, H. pylori 26695-infected and uninfected AGS cells (left) or human primary GECs (right) $(\mathrm{MOI}=100,24 \mathrm{~h})$ were analyzed by real-time PCR $(n=3)$ and western blot. d, e ETS1 mRNA and protein expression in H. pylori 11637-infected or H. pylori 26695 -infected AGS cells with different time points $(\mathrm{MOI}=100)(\mathbf{d})$ or at different $\mathrm{MOI}(24 \mathrm{~h})(\mathbf{e})$ were analyzed by real-time PCR $(n=3)$ and western blot. ${ }^{*} P<0.05,{ }^{*} P<0.01$.

RNA-seq analysis showed 76 genes were upregulated in H. pylori 11637-infected NC-transfected AGS cells and downregulated in H. pylori 11637-infected siETS1-transfected AGS cells (Fig. 4a). Among those genes, 40 proteincoding genes were founded (including ETS1) (Fig. 4a).
Gene-ontology functional analysis reveals that the ETS1 is primarily involved in biological processes including positive regulation of cell migration, positive regulation of cell motility, positive regulation of cellular component movement, positive regulation of inflammatory response, positive 

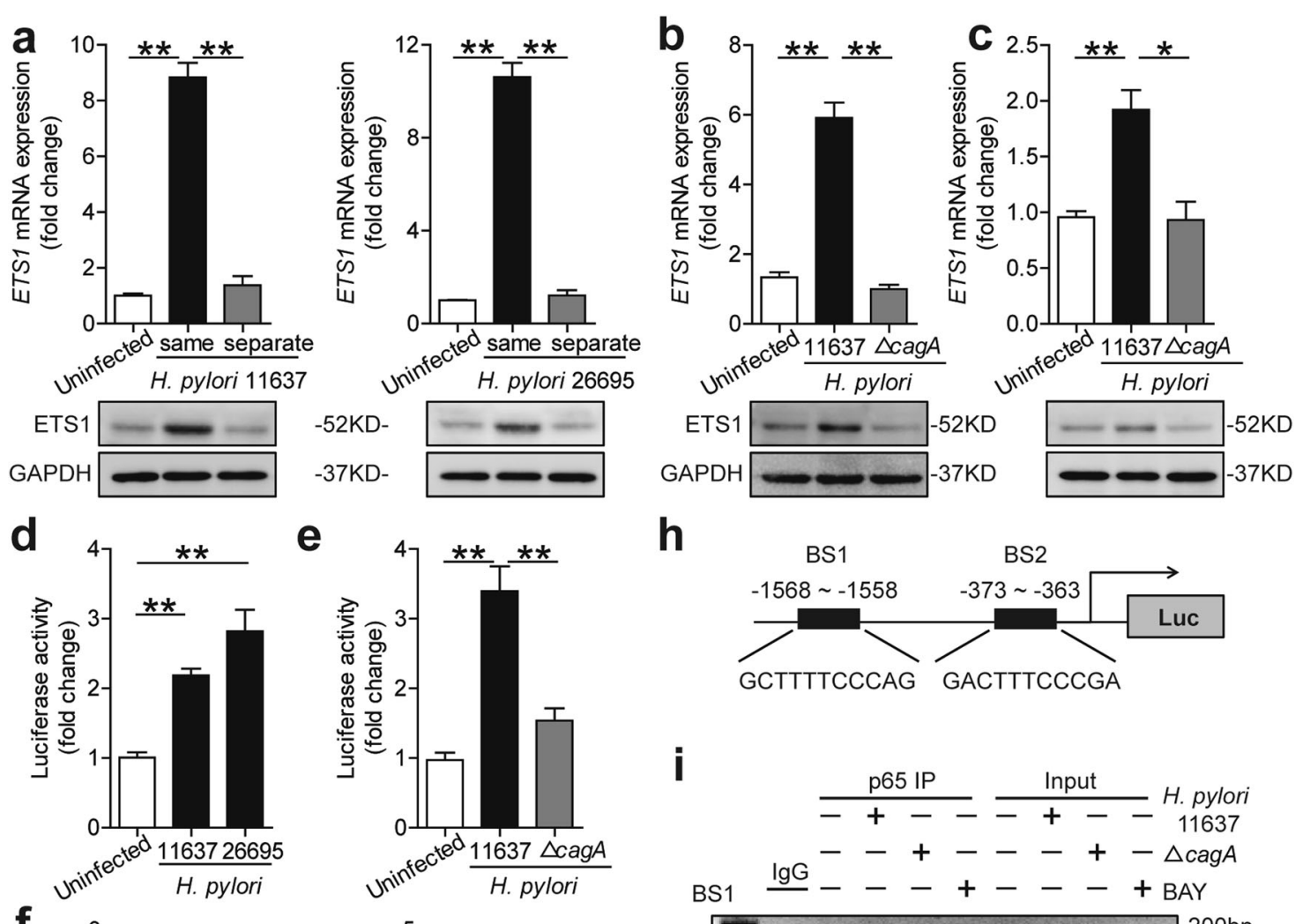

h

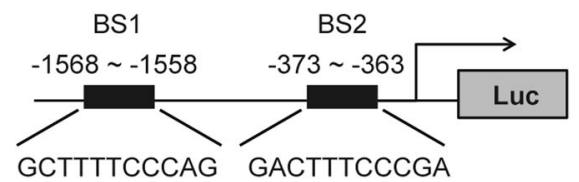

i
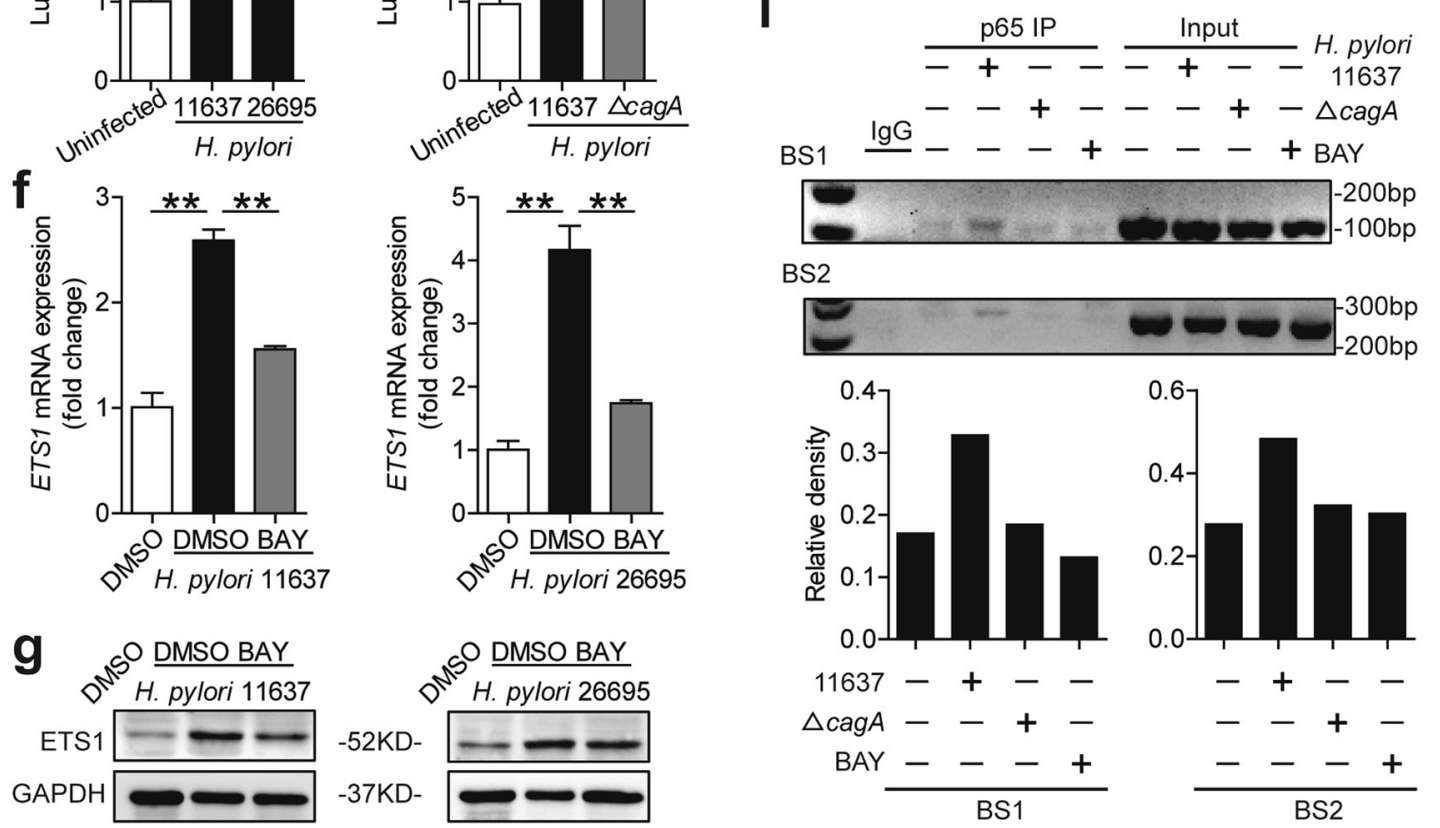

Fig. 2 The cagA activated NF-KB pathway-mediated ETS1 expression. a AGS cells were either infected or not by $H$. pylori $(\mathrm{MOI}=100)$ added in the same (lower) or separate (upper) chamber of a Transwell for $24 \mathrm{~h}$. ETS1 mRNA and protein expression levels were analyzed by real-time PCR ( $n=$ 3) and western blot. b, c ETS1 mRNA and protein expression in H. pylori 11637- or $\triangle$ cagA-infected and uninfected AGS cells (b) or human primary GECs $(\mathbf{c})(\mathrm{MOI}=100,24 \mathrm{~h})$ were analyzed by real-time PCR $(n=3)$ and western blot. $\mathbf{d}$ AGS cells were transfected with luciferase reporter constructs containing the ETS1-luc promoter for $4 \mathrm{~h}$. Luciferase activity was measured to assess promoter activity after H. pylori 11637 or H. pylori 26695 infection $(\mathrm{MOI}=100)$ for $24 \mathrm{~h}(n=3)$. e AGS cells were transfected with luciferase reporter constructs containing the ETS1-luc promoter for $4 \mathrm{~h}$. Luciferase activity was measured to assess promoter activity after $\mathrm{H}$. pylori 11637 or $\triangle$ cagA infection $(\mathrm{MOI}=100)$ for $24 \mathrm{~h}(n=3)$. f, $\mathbf{g}$ AGS cells were pretreated with BAY 11-7082 $(10 \mu \mathrm{M})$ and then infected with H. pylori 11637 or $\mathrm{H}$. pylori $26695(\mathrm{MOI}=100)$ for $24 \mathrm{~h}$. ETS1 mRNA and protein expression were analyzed by real-time PCR $(n=3)$ and western blot. $\mathbf{h}$ The potential binding sites for $\mathrm{p} 65$ in the promoter of ETS1. $\mathbf{i}$ Chromatin immunoprecipitation (ChIP) assay in AGS cells infected with H. pylori 11637 (cells pretreated or not with BAY 11-7082 before infection) or $\triangle$ cagA, followed by regular PCR with primers designed for p65 binding site of ETS1 promoter region. The density of the bands was quantified and is shown on the below, and the column diagrams showed the "antibody group/input group" according to the results. ${ }^{*} P<0.05,{ }^{* *} P<0.01$. 


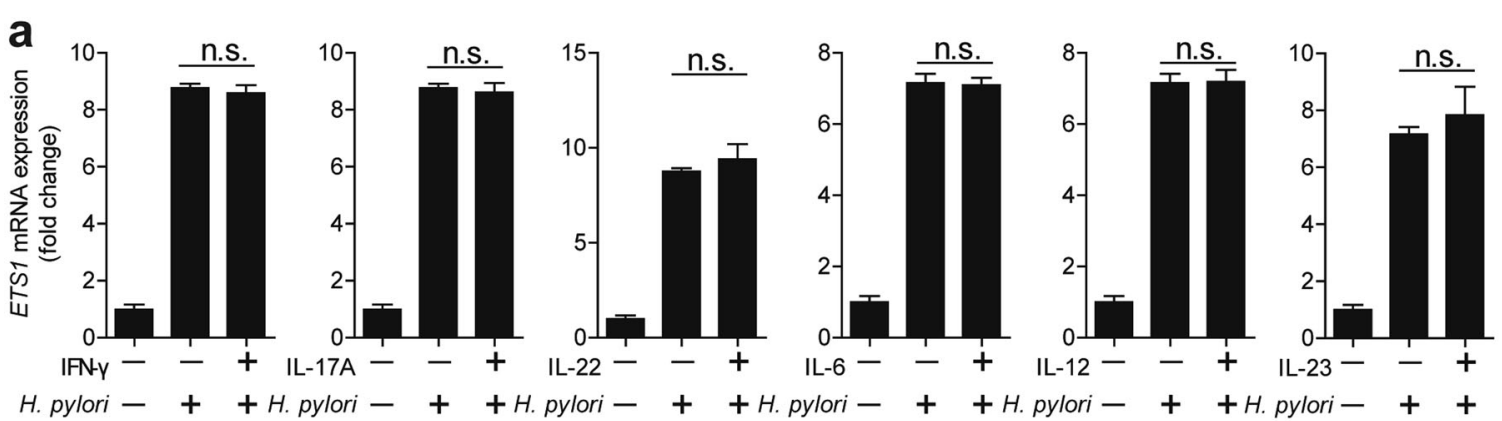

b

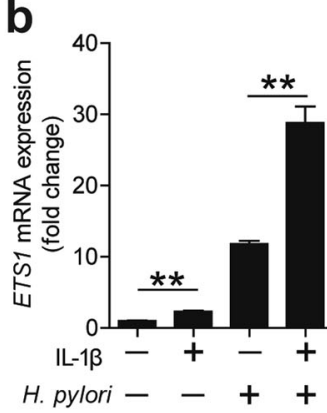

f

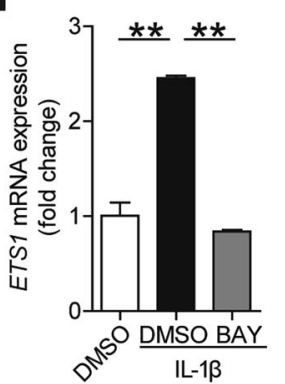

C
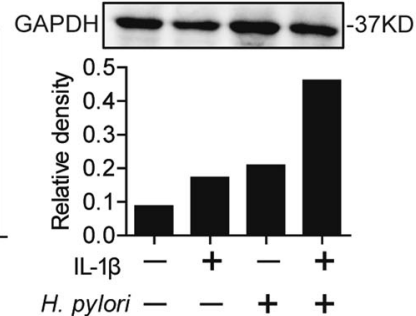

g
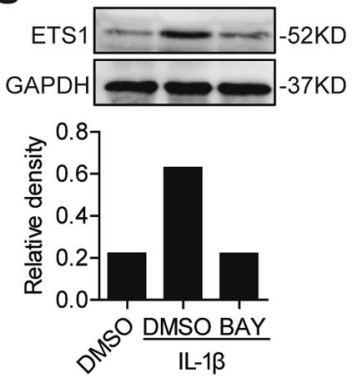

d

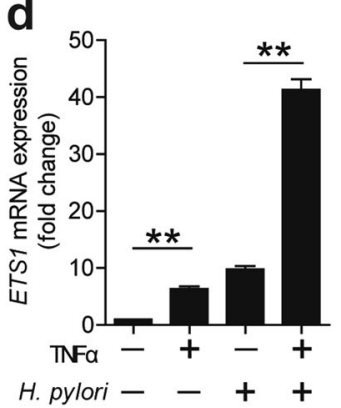

h

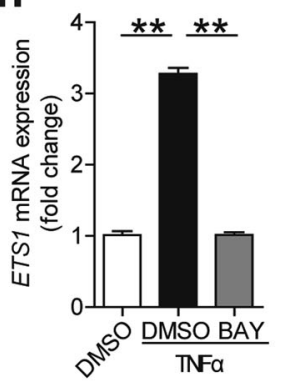

e

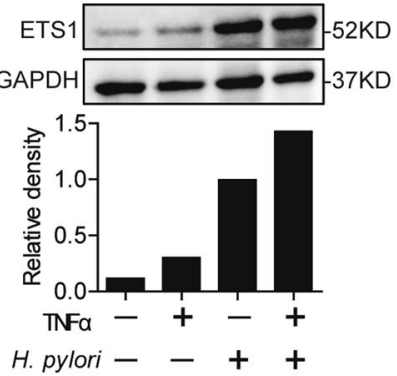

i
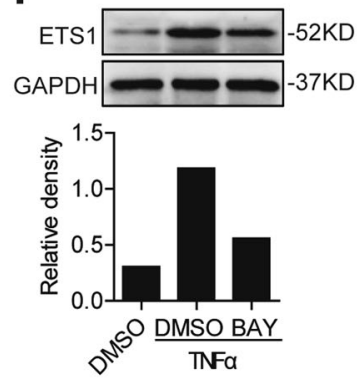

Fig. 3 IL-1 $\beta$ and TNFa have synergistic effects on ETS1 expression during $\boldsymbol{H}$. pylori infection. a ETS1 mRNA expression in AGS cells stimulated with H. pylori $11637(\mathrm{MOI}=100)$ and/or IFNy, IL-17A, IL-22, IL-6, IL-12, or IL-23 $(100 \mathrm{ng} / \mathrm{ml})(24 \mathrm{~h})$ were analyzed by real-time PCR $(n=3)$. The first three (IFNy, IL-17A, and IL-22) are the results of the same batch, and the last three (IL-6, IL-12, and IL-23) are the results of another same batch. $\mathbf{b}$, $\mathbf{d}$ ETS1 mRNA expression in AGS cells stimulated with H. pylori $11637(\mathrm{MOI}=100)$ and/or IL-1 $\beta(\mathbf{b})$ or TNFa $(\mathbf{d})(100 \mathrm{ng} / \mathrm{ml})(24 \mathrm{~h})$ were analyzed by real-time PCR $(n=3)$. c, e ETS1 protein expression in AGS cells stimulated with H. pylori $11637(\mathrm{MOI}=100)$ and/or IL-1 $\beta$ (c) or TNFa $(\mathbf{e})(100 \mathrm{ng} / \mathrm{ml})(24 \mathrm{~h}) \mathrm{were}$ analyzed by western blot. $\mathbf{f}, \mathbf{g}$ AGS cells were pretreated with BAY 11-7082 (10 $\mu \mathrm{M})$ and then treated with IL-1 $\beta$ for $24 \mathrm{~h}$. ETS1 mRNA (f) and protein $(\mathbf{g})$ expression were analyzed by real-time PCR $(n=3)$ and western blot. $\mathbf{h}$, i AGS cells were pretreated with BAY 11-7082 $(10 \mu \mathrm{M})$ and then treated with TNFa for $24 \mathrm{~h}$. ETS1 mRNA (h) and protein (i) expression were analyzed by real-time PCR $(n=3)$ and western blot. Quantized expression of proteins were analyzed by Image Lab software $(n=1)$. n.s. $P>0.05,{ }^{*} P<0.01$.

regulation of leukocyte migration, and positive regulation of locomotion (Fig. 4b). Collectively, these results suggest that ETS1 positively regulates inflammatory response during $H$. pylori infection may through regulate leukocyte migration and chemotaxis.

ETS1 is upregulated in mice and humans with $H$. pylori infection and its expression level is associated with the degree of gastric inflammation

To determine whether $H$. pylori infection increases ETS1 expression in gastric tissues, we conducted ELISA (serology test for $H$. pylori-specific antibodies) and PCR (test for $16 \mathrm{~S}$ rDNA) assessments to distinguish between the $H$. pylori-infected and uninfected individuals. We found ETS1 expression increased in gastric tissues from H. pylori-infected individuals (Fig. 5a). Then, an animal model was established by infecting mice with $H$. pylori 11637, and dynamic changes of Ets 1 mRNA expression were detected. The results showed that Ets1 mRNA expression reaching a peak of 12 weeks post-infection (Fig. 5b). Meanwhile, increased $I l-1 \beta$ and $t$ f $f \alpha$ were also found in our mouse model (SFig. 2a, b). Furthermore, ETS1/Ets1 protein was indeed increased in the gastric mucosa of $H$. pylori-infected patients and $H$. pyloriinfected mice, compared to those in uninfected patients or mice (Fig. 5e, f). Further, immunohistochemistry 

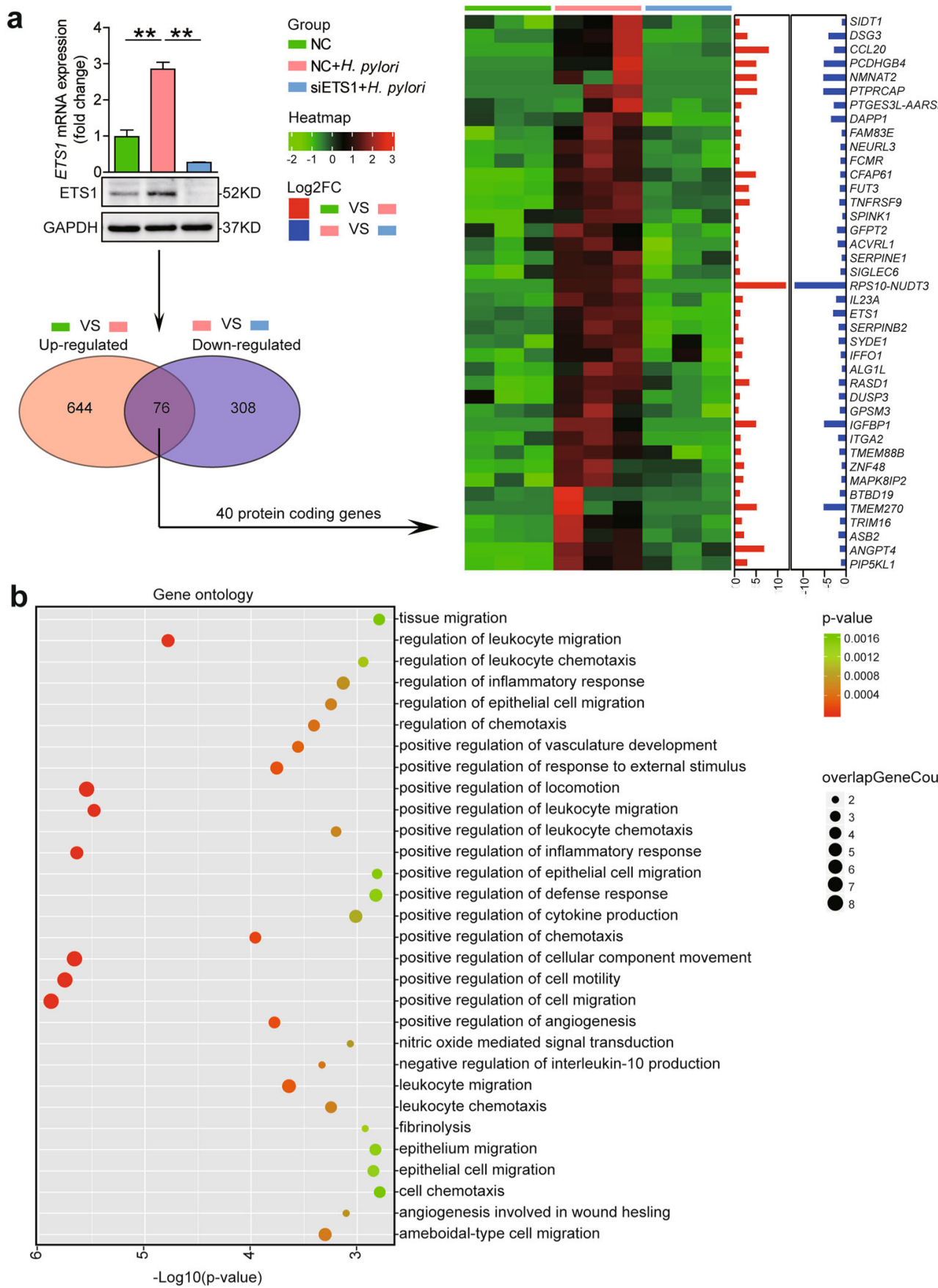

overlapGeneCount

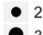

positive regulation of cellular component movement

.0016
.0012
.0008
.0004



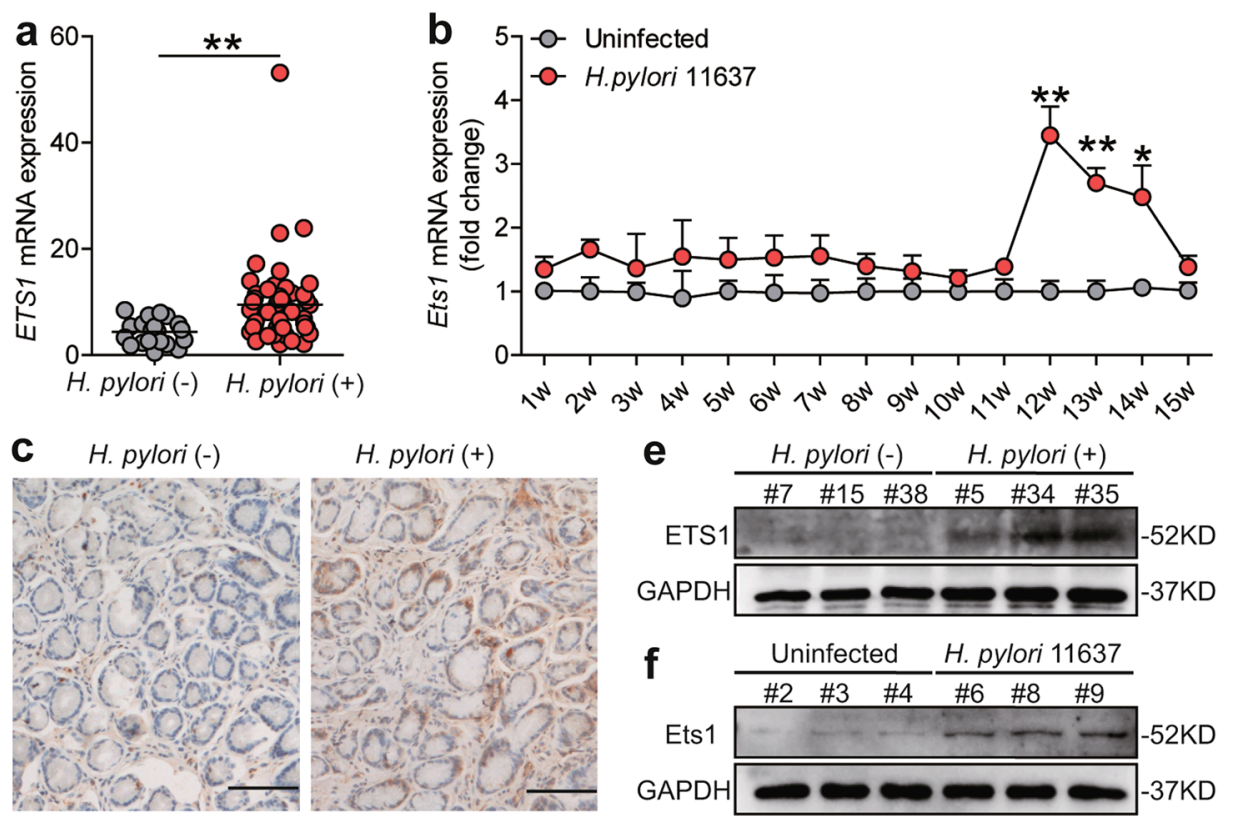

d Uninfected

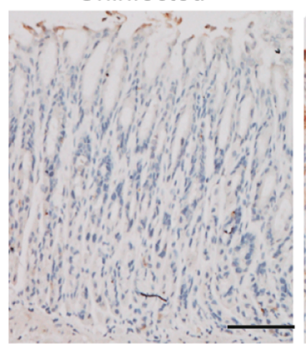

H. pylori 11637

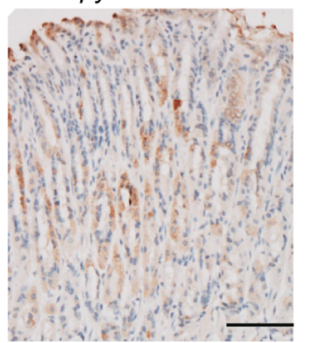

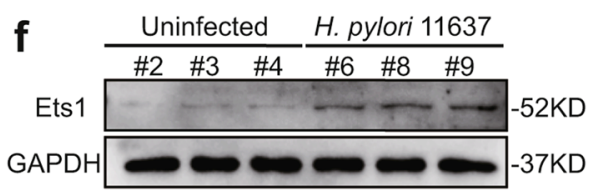

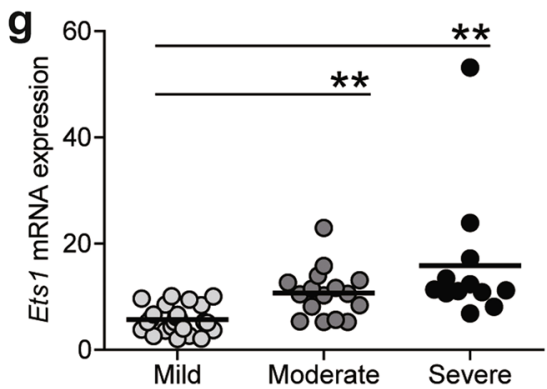

Fig. 5 ETS1 is upregulated in mice and human with H.pylori infection and associate with the degree of gastric inflammation. a ETS1 mRNA expression in gastric mucosa of H. pylori-infected (H. pylori $(+))(n=53)$ and uninfected donors $(H$. pylori $(-))(n=20)$ were compared. b Dynamic changes of Ets 1 mRNA expression in H. pylori 11637-infected and uninfected wild-type C57BL/6 mice $(n=5)$. c ETS1 protein expression in gastric mucosa of H. pylori (+) and H. pylori (-) was analyzed by immunohistochemistry staining. Scale bar: $100 \mu \mathrm{m}$. d Ets 1 protein expression in gastric mucosa of H. pylori 11637-infected and uninfected mice at 12 week p.i. was analyzed by immunohistochemical staining. Scale bar: $100 \mu \mathrm{m}$. e, f ETS1/ Ets1 protein in human gastric mucosa of H. pylori (+) and H. pylori (-) or in mice gastric mucosa of H. pylori 11637-infected and uninfected mice at 12 week p.i. was analyzed by western blot. $\mathbf{g}$ ETS1 mRNA expression in gastric mucosa of H. pylori $(+)$ patients with mild $(n=25)$, moderate $(n=16)$, severe $(n=12)$ inflammation were compared. ${ }^{*} P<0.05,{ }^{* *} P<0.01$.

\section{Discussion}

GECs infection by H. pylori result in multiple TF activation and increased inflammatory cytokine production, changes in biological characteristics, and ultimately oncogenic transformation ${ }^{6,24,25}$. ETS family is one of the largest families of evolutionarily conserved TFs, regulating critical cellular functions during cell homeostasis and contributing to tumor progression when perturbed ${ }^{26}$. In the present study, we screened ETS family gene expression in H. pyloriinfected mouse and human GECs, the results identified ETS1 as a key regulator. ETS1 was upregulated in mouse and human GECs with $H$. pylori infection, which was also confirmed by immunohistochemistry of biopsy specimens from patients. Notably, we also demonstrated that the ETS1 expression level in gastric mucosa was significantly correlated with the severity of gastric inflammation. Altogether, our findings demonstrate that ETS1 can be induced by $H$. pylori and ETS1 participates in proinflammatory response.

Previous studies have shown that the pathogenicity of H. pylori is largely attributed to virulence factor, cagA protein, which is a $120-140 \mathrm{kDa}$ protein and can be injected into GECs via the T4SS ${ }^{27}$. cagA has an important role in the proinflammatory response of GECs during H. pylori infection ${ }^{7,28}$. Using a cagA-KO mutant strain, we showed that ETS1 expression is largely cagA induced. It has been well established that cagA injected into host cells perturbs multiple central signaling pathways, especially NF- $k B$ signaling pathway ${ }^{29-31}$. The NF- $k B$ signaling pathway is a master regulator of inflammatory responses 
as it regulated in many cellular processes, including proliferation, angiogenesis, and even transformation ${ }^{32}$. Then, our findings demonstrated that cagA activated transcription factor NF-кB-p65 directly upregulate ETS1 expression.

H. pylori-infected gastric mucosa produces many proinflammatory cytokines, and the resultant inflammatory microenvironment could, in turn, modulate $H$. pylori-mediated gene expression in $\mathrm{GECs}^{22,23,33}$. In the present study, we found that IL- $1 \beta$ and TNF $\alpha$ have synergistic effects on $H$. pylori-mediated ETS1 expression through the NF- $\mathrm{KB}$ signaling pathway. The results revealed that ETS1 plays a pivotal role in $H$. pylori infection. To analyze the ETS1-controlled gene program, an RNA-seq assay was performed, and Gene-ontology functional analysis reveals that ETS1 positively regulate the inflammatory response. And we also found that ETS1 positively regulate leukocyte migration and chemotaxis. Therefore, ETS1 positively regulates inflammatory response may through regulate leukocyte migration and chemotaxis. However, the precise molecular mechanisms that ETS1 regulate leukocyte migration and chemotaxis also need further exploration.

In conclusion, our findings demonstrate the vital role of inflammation and $H$. pylori-mediated activation of the NF- $\mathrm{kB}$ pathway in ETS1 regulation in gastric gastritis. Meanwhile, ETS1 has a proinflammatory role in gastric gastritis was founded. Notably, these findings reveal that ETS1 may play a crucial role in the pathogenesis of $H$. pylori-associated gastritis.

\section{Materials and methods \\ H. pylori culture}

The cagA-positive H. pylori NCTC 11637 (ATCC 43504, called $H$. pylori 11637 in this article), cagA-KO mutant H. pylori NCTC 11637 (called $\triangle$ cagA in this article), and cagA-positive H. pylori 26695 (ATCC 700392) were cultured on brain-heart infusion plates containing 10\% rabbit blood at $37^{\circ} \mathrm{C}$ under microaerophilic conditions.

\section{Cell culture and stimulation}

The isolation of human primary GECs $\left(\mathrm{EpCAM}^{+}\right)$from tissues were performed as previously described ${ }^{34}$. Human primary GECs and AGS (ATCC) were cultured with DME/F12 (AGS) or RPMI 1640 (Human primary GECs) medium supplemented with $10 \%$ fetal bovine serum (FBS) at $37^{\circ} \mathrm{C}$ in $5 \% \mathrm{CO}_{2}$. Cells were stimulated with $H$. pylori 26695, H. pylori 11637, or $\triangle$ cagA at the indicated multiplicity of infection (MOI) and time. For H. pylori transwell infection ${ }^{35}$, cells were incubated in the presence of H. pylori that were added to the same (lower) or separate (upper) chamber of a $0.4 \mu \mathrm{m}$ transwell inserts for $24 \mathrm{~h}$. For cytokines stimulation, cells were stimulated with H. pylori $11637(\mathrm{MOI}=100)$ and/or $\mathrm{IFN}-\gamma(100 \mathrm{ng} / \mathrm{ml})$,
IL-17A (100 ng/ml), IL-22 (100 ng/ml), IL-6 (100 ng/ml), IL-12 (100 ng/ml), IL-23 (100 ng/ml), IL-1 $\beta(100 \mathrm{ng} / \mathrm{ml})$, or TNF $\alpha(100 \mathrm{ng} / \mathrm{ml})$ for $24 \mathrm{~h}$. For signal pathway inhibition experiments, cells were pretreated with BAY 11-7082 $(10 \mu \mathrm{M})$ or DMSO for $2 \mathrm{~h}$, then, infected with H. pylori 11637 or 26695 for $24 \mathrm{~h}$. After co-culture, cells were collected for real-time PCR and western blot.

\section{Real-time PCR analysis}

RNAiso Plus extracted total RNA from biopsy specimens, mouse gastric tissues and cultured cells were reversetranscribed to cDNA by PrimeScript ${ }^{\mathrm{TM}} \mathrm{RT}$ reagent Kit. For mouse samples, expression levels were normalized to $\beta$-actin. And for human samples, GAPDH served as the normalizer. Real-time PCR was performed on the CFX96 (Bio-Rad, Hercules, USA) with the SYBO Green Real-time PCR Master Mix according to the manufacturer's specifications. The relative gene expression was expressed as fold change calculated by the $\Delta \Delta \mathrm{Ct}$ method. The sequences of specific primers are presented in Supplementary Table 3.

\section{Western blot}

In brief, equivalent amounts of cell or tissue lysates were resolved in 10\% SDS-PAGE gels, and proteins were then transferred onto PVDF membranes and western blots were performed. 5\% BSA was used for blocking the PVDF membranes. In turn, membranes were incubated with specific antibody $(\mathrm{Ab})$. This was followed by incubation with HRP-conjugated secondary Abs. Detected proteins were visualized by using ECL chemiluminescence kit. Protein levels were quantified using the Image Lab software, version 3.0 (Bio-Rad, USA).

\section{Luciferase reporter assay}

Promoter constructs containing the region from -2000 to 0 of the ETS1 gene were amplified from human genomic DNA by PCR (constructed by Sangon Biotech, Shanghai, China). The promoter sequences $(-2000 / 0)$ (Supplementary Table 2) were obtained from The Eukaryotic Promoter Database (EPD, https://epd.epfl.ch// index.php). The amplified sequences were cloned into the NheI and HindIII sites of the pGL3-basic vector (Promega, Madison, USA). Cells were transfected with pGL3ETS1 and internal control pRL-TK (Promega, Madison, USA) by using Lipofectamine 2000 according to the manufacturer's protocol for $4 \mathrm{~h}$. Next, cells were infected with $H$. pylori 26695,11637 or $\Delta$ cagA $(\mathrm{MOI}=100)$. The luciferase activities in the cell lysates were measured using the Dual-Luciferase Reporter assay after $24 \mathrm{~h}$ following the manufacturer's protocol.

\section{Chromatin immunoprecipitation (ChIP) assay}

Cells were infected with $H$. pylori 11637 (cells pretreated with or without BAY 11-7082) or $\triangle$ cagA for $18 \mathrm{~h}$. Then, the 
EZ-Magna ChIP ${ }^{\mathrm{TM}}$ A/G Chromatin Immunoprecipitation Kit was used following the manufacturer's protocol. The sequences of primers for ETS1 promoter were as follows: BS1, forward, 5'-GTGGTTCATTTGGACGTGTAAATGT3'; reverse, 5'-TGCAATCCAGCTGTGTTTCCA-3' (product length $141 \mathrm{bp}$ ); BS2, forward, 5'-AGGACACGGGCT CACGAATC-3'; reverse, 5'-GGAGGAGCAGTGCGTGG AG-3' (product length $293 \mathrm{bp}$ ). The band densities were quantified using the Image J software $(\mathrm{NIH}$, Bethesda, $\mathrm{MD}$, USA). The column diagrams showed the "antibody group/ input group" according to the results.

\section{Small interfering RNA (siRNA) transfection}

siRNA targeting ETS1 and control siRNA were purchased from GenePharma (Shanghai, China). The ETS1-targeting siRNA (siETS1) sequences were: sense, 5'-GCAGCCAGUC AUCUUUCAATT-3', and antisense, 5'-UUGAAAGAUGA CUGGCUGCTT-3'. The control siRNA (Negative control (NC)) sequences were: sense, 5'-UUCUCCGAACGUGUCA CGUTT-3', and antisense, 5'-ACGUGACACGUUCGGAG AATT-3'. About 50\% confluent monolayers were washed and incubated in serum- and antibiotic-free medium. All siRNA transfections were performed using Lipofectamine 2000 according to the manufacturer's protocol.

\section{RNA-seq}

RNAiso Plus was used for extracting total RNA from cultured cells. The RNA quality was measured using Agilent 4200 TapeStation. Then, RNA quantity was measured using Qubit 2.0 Fluorometer. RNA samples were sent to Genminix Informatic Ltd. (Shanghai, China) for mRNA sequencing on Illumina Hiseq platform (Illumina, San Diego, California, USA) with 6 Gbps. Gene-ontology functional analysis was used to categorize the functions of differentially expressed target genes.

\section{H. pylori infection of mice}

Female wild-type (WT) C57BL/6 mice were purchased from the Experimental Animal Centre of the Third Military Medical University. For infecting mice, $H$. pylori were amplified in Brucella broth with $5 \%$ fetal bovine serum with gentle shaking at $37^{\circ} \mathrm{C}$ under microaerobic conditions. Twenty hour later, live bacteria were collected and adjusted to $10^{9}$ colony forming units (CFU)/mL. Mice were fasted overnight and orogastrically inoculated twice at a 1-day interval with $5 \times 10^{8} \mathrm{CFU}$ bacteria. Five mice per group per time point. All breeding and experiments were reviewed and approved by the Animal Ethical and Experimental Committee of Third Military Medical University.

\section{Immunohistochemistry}

Mouse and human gastric tissue sections were deparaffinized, rehydrated and boiled in citrate buffer. Sections were incubated with primary anti-ETS1 Ab overnight at $4{ }^{\circ} \mathrm{C}$. The primary Ab was detected using a biotinylated secondary $\mathrm{Ab}$ followed by incubation with horseradish peroxidase-conjugated avidin to form avidin-biotin complexes according to the manufacturer's protocol. Slides were then stained with DAB, counterstained with haematoxylin and photomicrographs captured using a bright field microscope (Nikon Eclipse 80i; Nikon, Tokyo, Japan).

\section{Antibodies and other reagents \\ Details are available in the Supplementary Table 4.}

\section{Ethical statement, patients, and specimens}

The gastric biopsy specimens were gathered from 73 patients $(53 \mathrm{H}$. pylori-infected patients and 20 uninfected volunteers) who underwent upper esophagogastroduodenoscopy for dyspeptic symptoms at Xinqiao Hospital. The grade of gastritis (mild, moderate and severe) was based on the density of infiltrating mononuclear and polymorphonuclear cells according to the updated Sydney System ${ }^{36}$. The histopathological analysis was carried out by two experienced histopathologists. All experimental procedures were approved by the Third Military Medical University's human ethics committee and written informed consent obtained from each patient. The clinical characteristics of all patients were described in Supplementary Table 5.

\section{Statistical analysis}

Quantitative data are presented as the mean \pm SEM. Generally, Student $t$-test was used to analyze the differences between two groups; however, when the variances differed, the Mann-Whitney $U$ test was used. All data were analyzed using the GraphPad Prism software version 5.0. For RNA-seq analysis, the differentially expressed genes were selected as having more than two-fold difference between their geometrical mean expression in the compared groups and a statistically significant $P$-value $(<0.05)$ by analysis of DEseq2. Statistical significance was defined when $P<0.05$.

\footnotetext{
Acknowledgements

We thank Shi-ming Yang (Department of Gastroenterology, XinQiao Hospital, Third Military Medical University, Chongqing, China) for collecting gastric biopsy specimens from $\mathrm{H}$. pylori-infected and uninfected patients. We thank Yi Zhang (Genminix Informatics Co., Shanghai, China) and Ming-song Feng (Genminix Informatics Co., Shanghai, China) for technical assistance and bioinformatics support. This work was supported by grant of National Natural Science Foundation of China (81870394), Chongqing National Science Fund for Distinguished Young Scholars (cstc2019jcyjjqX0003), National Key Research and Development Program of China (2016YFC1302200), and Science Innovation Capacity Promotion Project of Army Medical University (2019XQY03).
}

\section{Author details}

${ }^{1}$ National Engineering Research Centre of Immunological Products, Department of Microbiology and Biochemical Pharmacy, College of Pharmacy and Laboratory Medicine, Third Military Medical University, Chongqing, China. 
${ }^{2}$ The 988 Hospital of PLA, Henan, Zhengzhou, China. ${ }^{3}$ La Trobe Institute of Molecular Science, La Trobe University, Bundoora, VIC 3086, Australia. ${ }^{4}$ Department of Hepatobiliary Surgery, Xinqiao Hospital, Third Military Medical University, Chongaing, China

\section{Conflict of interest}

The authors declare that they have no conflict of interest.

\section{Publisher's note}

Springer Nature remains neutral with regard to jurisdictional claims in published maps and institutional affiliations.

Supplementary Information accompanies this paper at (https://doi.org/ 10.1038/s41419-020-2705-8).

Received: 18 December 2019 Revised: 4 June 2020 Accepted: 19 June 2020 Published online: 01 July 2020

\section{References}

1. Hooi, J. K. Y. et al. Global prevalence of Helicobacter pylori infection: systematic review and meta-analysis. Gastroenterology 153, 420-429 (2017).

2. Jackson, L. et al. A population-based epidemiologic study of Helicobacter pylori infection and its association with systemic inflammation. Helicobacter 14, 108-113 (2009).

3. Mejias-Luque, R. et al. Lymphotoxin beta receptor signalling executes Helicobacter pylori-driven gastric inflammation in a T4SS-dependent manner. Gut 66, 1369-1381 (2017).

4. Morey, P. et al. Helicobacter pylori depletes cholesterol in gastric glands to prevent interferon gamma signaling and Escape the Inflammatory Response. Gastroenterology 154, 1391-1404 e1399 (2018).

5. Papavassiliou, K. A. \& Papavassiliou, A. G. Transcription factor drug targets. J. Cell. Biochem. 117, 2693-2696 (2016)

6. Menheniott, T. R., Judd, L. M. \& Giraud, A. S. STAT3: a critical component in the response to Helicobacter pylori infection. Cell. Microbiol. 17, 1570-1582 (2015).

7. Wang, F., Meng, W., Wang, B. \& Qiao, L. Helicobacter pylori-induced gastric inflammation and gastric cancer. Cancer Lett. 345, 196-202 (2014).

8. Suzuki, M. et al. Helicobacter pylori CagA phosphorylation-independent function in epithelial proliferation and inflammation. Cell Host Microbe $\mathbf{5}$ 23-34 (2009).

9. Zook, E. C. et al. The ETS1 transcription factor is required for the development and cytokine-induced expansion of ILC2. J. Exp. Med. 213, 687-696 (2016).

10. Lee, C. G. et al. Ets1 suppresses atopic dermatitis by suppressing pathogenic T cell responses. JCl Insight 4, e124202. https://doi.org/10.1172/jci.insight.124202 (2019).

11. Leng, R. X. et al. The dual nature of Ets-1: focus to the pathogenesis of systemic lupus erythematosus. Autoimmun. Rev. 10, 439-443 (2011).

12. Dittmer, J. The role of the transcription factor Ets1 in carcinoma. Semin. Cancer Biol. 35, 20-38 (2015).

13. Garrett-Sinha, L. A. Review of Ets1 structure, function, and roles in immunity. Cell. Mol. life Sci. 70, 3375-3390 (2013).

14. Yu, Y. et al. Ets1 as a marker of malignant potential in gastric carcinoma. World J. Gastroenterol. 9, 2154-2159 (2003).

15. Nakayama, T. et al. Expression of the Ets-1 proto-oncogene in human gastric carcinoma: correlation with tumor invasion. Am. J. Pathol. 149, 1931-1939 (1996).
16. Tsutsumi, S. et al. Expression of Ets-1 angiogenesis-related protein in gastric cancer. Cancer Lett. 160, 45-50 (2000).

17. Chen, W. et al. Disparities by province, age, and sex in site-specific cancer burden attributable to 23 potentially modifiable risk factors in China: a comparative risk assessment. Lancet Glob. Health 7, e257-e269 (2019).

18. Amieva, M. \& Peek, R. M. Jr. Pathobiology of Helicobacter pylori-induced gastric cancer. Gastroenterology 150, 64-78 (2016).

19. Giannakis, M., Chen, S. L., Karam, S. M., Engstrand, L. \& Gordon, J. I. Helicobacter pylori evolution during progression from chronic atrophic gastritis to gastric cancer and its impact on gastric stem cells. Proc. Natl Acad. Sci. USA 105, 4358-4363 (2008)

20. Messeguer, $X$. et al. PROMO: detection of known transcription regulatory elements using species-tailored searches. Bioinformatics $\mathbf{1 8}$ 333-334 (2002)

21. Farré, D. et al. Identification of patterns in biological sequences at the ALGGEN server: PROMO and MALGEN. Nucleic Acids Res. 31, 3651-3653 (2003).

22. Lina, T. T. et al. CagA-dependent downregulation of $\mathrm{B} 7-\mathrm{H} 2$ expression on gastric mucosa and inhibition of Th17 responses during Helicobacter pylori infection. J. Immunol. 191, 3838-3846 (2013).

23. LV, Y. P. et al. Helicobacter pylori-induced matrix metallopeptidase-10 promotes gastric bacterial colonization and gastritis. Sci. Adv. 5, eaau6547 (2019).

24. Posselt, G., Backert, S. \& Wessler, S. The functional interplay of Helicobacter pylori factors with gastric epithelial cells induces a multi-step process in pathogenesis. Cell Commun. Signal. 11, 77 (2013).

25. Zhu, S. et al. Helicobacter pylori-induced cell death is counteracted by NFkappaB-mediated transcription of DARPP-32. Gut 66, 761-762 (2017).

26. Findlay, V. J., LaRue, A. C., Turner, D. P., Watson, P. M. \& Watson, D. K. Understanding the role of ETS-mediated gene regulation in complex biological processes. Adv. Cancer Res. 119, 1-61 (2013).

27. Yamaoka, Y. Mechanisms of disease: Helicobacter pylori virulence factors. Nature reviews. Gastroenterol. Hepatol. 7, 629-641 (2010).

28. Zhuang, Y. et al. A pro-inflammatory role for Th22 cells in Helicobacter pyloriassociated gastritis. Gut 64, 1368-1378 (2015).

29. Yong, $X$. et al. Helicobacter pylori virulence factor CagA promotes tumorigenesis of gastric cancer via multiple signaling pathways. Cell Commun. Signal. 13, 30 (2015).

30. Keates, S., Hitti, Y. S., Upton, M. \& Kelly, C. P. Helicobacter pylori infection activates NF-kappa B in gastric epithelial cells. Gastroenterology 113, 1099-1109 (1997)

31. Kang, M. J. et al. NF-kappaB activates transcription of the RNA-binding factor HuR, via PI3K-AKT signaling, to promote gastric tumorigenesis. Gastroenterology 135, 2030-2042 (2008).

32. DiDonato, J. A., Mercurio, F. \& Karin, M. NF-kappaB and the link between inflammation and cancer. Immunol. Rev. 246, 379-400 (2012).

33. Bockerstett, K. A. \& DiPaolo, R. J. Regulation of gastric carcinogenesis by inflammatory cytokines. Cell. Mol. Gastroenterol. Hepatol. 4, 47-53 (2017).

34. Teng, Y. et al. Helicobacter pylori-downregulated tumor necrosis factor receptor-associated protein 1 mediates apoptosis of human gastric epithelial cells. J. Cell. Physiol. https://doi.org/10.1002/jcp.28223 (2019).

35. Whitmore, L. C., Weems, M. N. \& Allen, L. H. Cutting edge: Helicobacter pylori induces nuclear hypersegmentation and subtype differentiation of human neutrophils in vitro. J. Immunol. 198, 1793-1797 (2017).

36. Dixon, M. F., Genta, R. M., Yardley, J. H. \& Correa, P. Classification and grading of gastritis. The updated Sydney System. International Workshop on the Histopathology of Gastritis, Houston 1994. Am. J. Surg. Pathol. 20, 1161-1181 (1996). 